\title{
ESCALA DE FACTORES ESTRUCTURALES DE LA CLASE: UNA MEDIDA PARA EVALUAR CARACTERÍSTICAS DE LA CLASE RELACIONADAS CON LAS METAS ACADÉMICAS DE LOS ESTUDIANTES'
}

C ARLOS CALDERÓN ${ }_{2}$ (UNIVERSIDAD AUTÓNOMA DE MADRID, Madrid, España).

GIULIA CASU (UNIVERSITÀ DI BOLOGNA, Bologna, Italia).

\begin{abstract}
RESUMEN
El presente estudio presenta los resultados psicométricos de la escala para evaluar los factores estructurales de la clase propuestos por Ames (1992) relacionados con la adquisición por parte de los estudiantes de Metas de Dominio. La muestra estuvo compuesta por 275 estudiantes universitarios de $1^{\circ}$ a $4^{0}$ años de carreras de Ingeniería y Periodismo. El instrumento obtuvo una alta consistencia interna y el análisis factorial mostró una estructura coherente con la propuesta teórica. Finalmente la existencia de correlaciones significativas con la orientación de metas de Dominio se transforma en importantes evidencias de validez convergente
\end{abstract}

PALABRAS CLAVE: Metas de aprendizaje; factores estructurales de la clase.

\section{ABSTRACT}

This study presents the results of psychometric scale to assess the structural factors of classroom proposed by Ames (1992) related to the acquisition by students of mastery goals. The sample of 275 college students from lst to 4th year of Engineering and Journalism. The instrument had a high internal consistency and factor analysis showed a structure consistent with the theoretical proposal. Finally, the existence of significant correlations with mastery goals orientation becomes strong evidence of convergent validity

KEY WORDS: Learning goals; structural factors of classroom.

1. Este estudio se realizó gracias al apoyo financiero de la Dirección General de Docencia de la Universidad Católica del Norte y el patrocinio del Centro de Innovación Metodológica y Tecnológica CIMET, Universidad Católica del Norte .

2. Comunicación con los autores. Email: ccalderon@ucn.cl 


\section{INTRODUCCIÓN}

Dentro del ámbito académico se han abordado un sin numero de variables relacionadas con el rendimiento de los estudiantes tales como, percepción de sí mismo y de la tarea, actitudes, intereses, expectativas, represtaciones, etc. (González-Cabanach y cols, 1996). A causa de la importancia que tiene la motivación sobre el aprendizaje, gran número de estudios se han centrado en los factores que influyen sobre ella.

En este contexto, una de las líneas de investigación en psicología educacional más fructíferas en los últimos años está relacionada con el constructo Orientación de Metas. Los modelos desarrollados en esta línea han mostrado que la meta es una variables de elevada importancia a la hora de explicar la conducta motivada (Navas y Sampascual, 2008), y corresponde al marco de referencia más utilizado en la actualidad para abordar la motivación hacia aprendizaje por parte de los estudiantes (Valle y cols., 2006).

La Orientación de meta hace referencia al propósito que se plantea la persona al momento de abordar una tarea, la cual abarca tanto los fines que persigue, como también los criterios con los cuales el sujeto evalúa su desempeño en ella y por los cuales juzga su éxito o fracaso (Pintrich, 2000).

La Meta corresponde a un patrón integrado de creencias, afectos y/o atribuciones que dirigen las intenciones conductuales (Wainer, 1986), y configurarían variadas formas de aproximación, compromiso y respuesta ante el logro de la tarea (Ames, 1992b, Dweck y Laget, 1988).

A pesar que existe un grupo importante de autores que define de forma distinta los tipos de meta adquiridas los sujetos (González y cols, 1996), es posible observar la existencia de dos importantes grupos de orientaciones de meta (ver tabla 1).

TABLA 1.

Tipos de orientación de metas

\begin{tabular}{llll}
\hline \multicolumn{5}{c}{ Orientación de Metas } & & \\
\hline Dwek, & Metas Centradas & Metas & de \\
1986 & en el Aprendizaje & Ejecución & \\
Nichols, & Metas Centradas & Metas Centradas \\
1984 & en la Tarea & en el Yo & \\
Ames, & Metas de Dominio & Metas & de \\
1992 & & Rendimiento & \\
\hline
\end{tabular}

La primera de ellas, la Orientación de Metas de Dominio (en la nomenclatura de Ames), se caracteriza por la búsqueda del aprendizaje y dominio de la tarea, el desarrollo de habilidades, y la búsqueda de la mejora y la superación personal. Contraria a ella, la segunda orientación de meta, orientada al Rendimiento, se caracteriza por la necesidad de demostrar la competencia 0 habilidad, obtener aprobación de terceros, evitar los juicios negativos u obtener beneficios secundarios (Navas y Sampascual, 2008). Esta tendría un carácter más extrínseco.

Como es de suponer, la adquisición por parte del estudiante de tal o cual orientación de meta influye directamente en la conducta desplegada por el sujeto a la hora de enfrentar una situación de aprendizaje. Los sujetos con metas de Rendimiento desarrollaran conductas centradas en la consecución de metas extrínsecas, como obtención de notas, evitación de castigos 0 aprobación de pares, es el llamado patrón de indefensión (helpness). Para los sujetos los fracasos sueles provocar una percepción de baja capacidad, siendo interpretados como una falta de habilidad o competencia, lo que se traduce en falta de implicación en la tarea, baja persistencia, y falta de interés en la búsqueda de nuevas estrategias que permitan resolver las dificultades (Navas, González y Torregrosa, 2002). Este patrón no esta relacionado con el interés intrínseco, sino que lleva al sujeto a la búsqueda de tareas fáciles y la evitación de 
tareas difíciles o del riesgo que puedan involucrar.

En cambio, las metas orientadas al Dominio se centran en el desarrollo de capacidades 0 habilidades. Los sujetos buscan estrategias de autorregulación que les ayude resolver dificultades, y valoran el aprendizaje, dedicando un mayor esfuerzo y atención a tareas nuevas y/o difíciles (Ames, 1984; Dweck y Leggett, 1989). El propósito final es el dominio de la tarea y de la habilidad.

Aun que ambos tipos de metas pueden influir de manera positiva en el rendimiento del estudiante, la orientación de meta de Dominio resulta mucho más adaptativa (González-Cabanach et Al, 1996). Un gran número de investigaciones indican que los estudiantes muestran un patrón motivacional mucho más positivo cuando ellos están orientados hacia metas de Dominio (Meece, Anderman y Anderman, 2006). Logran persistir más en tareas de gran dificulta (Elliot y Dweck, 1988), reportan altos niveles de involucramiento (Harackievicz et. Al. 2000), altos niveles de esfuerzo y persistencia (Grant y Dweck, 2003; Wolters, 2004), y usan estrategias de aprendizaje mas eficaces para la comprensión de conceptos y la recuperación de información (Ames y Archer, Grant y Dweck, 2003; Miller et. Al. 1996; Wolters, 2004).

Es así que las metas que se plantee el sujeto determinan su motivación frente a la situación de aprendizaje. En la medida en que esté orientado al dominio o aprendizaje, logrará desplegar una conducta más adaptativa frente a las dificultades que la tarea presente.

Ahora bien, que un estudiante adquiera tal o cual meta depende tanto de factores individuales como situacionales. Aun que las variables individuales juegan un importante papel en la adquisición de metas, no se pueden dejar de lado las variables situacionales cercanas al sujeto (González-Cabanach y cols, 1996), especialmente por que revisten particular importancia en la intervención educativa en ambientes de aprendizaje.

Uno de los principales aportes de la perspectiva sociocognitiva en esta línea ha sido la importancia otorgada a los factores estructurales de la clase. Desde esta perspectiva se asume que las metas surgen tanto del individuo como del contexto en el cual se desenvuelve (Navas y Sampascual, 2008). De hecho, el proceso de enseñanzaaprendizaje esta inserto en un contexto social determinado por expectativas y normas que regulan la dinámica de la clase (Wentzel, 2000). Es por tanto fundamental en la orientación de meta que el estudiante adquiere, la percepción que este tiene de determinados aspectos del ambiente en el cual se desarrolla este proceso.

Ames (1992a), logra distinguir los elementos más importantes relacionados con la estructura de la clase que promueven en los estudiantes la adquisición de metas de dominio, así como también, como estos elementos influyen en la forma de involucrarse en el aprendizaje. Es así que plantea tres dimensiones importantes de la estructura de la clase que influyen en las metas de los estudiantes: [1] Tareas y actividades de aprendizaje, [2] Sistema de Evaluación y Recompensa, y [3] Distribución de poder y Autoridad.

Tareas y actividades de aprendizaje. Un elemento central del aprendizaje en la sala de clases es el diseño de las tareas y actividades de aprendizaje. La percepción que el estudiante tiene de ellas tiene una enorme influencia en la manera de involucrarse en su propio aprendizaje. La naturaleza y características de la actividad son utilizadas por el estudiante para realizar juicios acerca de su habilidad y competencia para abordarla, el esfuerzo que utilizará en resolverla, y la posterior satisfacción que le proporcionará. 
Un importante número de investigaciones señalan que la variedad y diversidad de actividades promueve interés en el aprendizaje y la orientación al Dominio (Ames, 1992a). Además, es necesario que las actividades de aprendizaje estén centradas en aspectos significativos, ofrezcan retos y desafíos razonables, fomenten la autorregulación y el establecimiento de metas auto-referentes, y potencien la utilización y el desarrollo de distintas estrategias de aprendizaje (González-Cabanach y cols. 1996).

Sistema de evaluación y recompensa. Según Ames (1996a), la forma en la cual el estudiante es evaluado es uno de los factores más relevantes que afectan la motivación del estudiante. Los standards, criterios y métodos, así como el contenido, la frecuencia y la retroalimentación, son algunos de elemento a considerar.

De acuerdo con Ames, la evaluación debe estar centrada en el progreso y mejora de las habilidades particulares del estudiante, reconocer su esfuerzo, retroalimentar de manera individual, dando oportunidades para progresar, y fomentando la visión de los errores como oportunidades de aprendizaje y parte constitutiva del proceso de aprendizaje.

Distribución de poder y Autoridad. La capacidad de orientar a los estudiantes hacia la autonomía o el nivel en que el profesor lo involucra en las decisiones de la clase esta relacionado con un patrón motivacional positivo, corroborado en numerosos estudios (Ames, 1996a).

Dar espacio para el desarrollo de responsabilidad y autonomía, promover el la mejora de competencias de autorregulación y control, son estrategias instruccionales que favorecen la adquisición de metas de dominio por parte del estudiantes (González-Cabanach y cols. 1996).
Dentro de esta dimensión uno de los factores más significativos, que afecta directamente el involucramiento de los estudiantes en su aprendizaje es la percepción de control. Cuando el estudiante se siente independiente y autónomo, valora mucho más el uso de estrategias de aprendizaje eficaces (Nolen y Haladyna, 1990b, en Ames, 1996a). La comprensión y el aprendizaje parecen ser facilitados por condiciones ambientales que minimizan la percepción de control externo y al mismo tiempo focalizan al estudiante en la tarea (Ames, 1992a). La posibilidad que tenga el estudiante de opinar y decidir en la selección de tareas, materiales, métodos de aprendizaje, en síntesis, tomar decisiones acerca de su propio proceso de aprendizaje, contribuye significativamente a la percepción de control, aumentando su nivel de interés e involucramiento.

Aunque cada clase corresponde a un contexto particular, con actores y dinámicas particulares, las estructuras anteriormente descritas se transforman en un marco comprensivo general, que puede ayudar al profesor a focalizar intervenciones y diseñar estrategias que persigan la orientación de dominio de sus estudiantes.

Con este propósito Ames (1992b, en Meece, Anderman y Anderman, 2005) desarrolla el TARGET system (Sistema de Metas). Modelo de evaluación que promueve la identificación las prácticas asociadas con una orientación de dominio y/o rendimiento del estudiante en la clase. Estas prácticas son estrategias instruccionales agrupadas en una serie de áreas, alguna de ellas son: Tareas y actividades de aprendizaje ("Task assignments"), Relaciones de Autoridad ("Authority relations"), Sistema de Refuerzos ("Recognition system"), y Practicas de Evaluación ("Evaluation practices").

A partir de la estructura planteada es que nos hemos propuesto la elaboración de una medida de auto-reporte que nos ayude a 
evaluar el nivel en que estos factores estructurales se encuentran relacionados con la adquisición de metas de dominio por parte del estudiante. Lo anterior con dos propósitos fundamentales. Por un lado un instrumento de esta naturaleza contribuirá de manera significativa al estudio de la motivación al aprendizaje particularmente desde la perspectiva sociocognitiva, incorporando variables del contexto de la sala de clase 0 ambiente de aprendizaje, y por otro, desde un punto de vista más aplicado, este instrumento puede transformarse en una herramienta con la cual monitorear y evaluar intervenciones instruccionales que puedan promover en los estudiantes la adquisición de Metas de Dominio.

\section{MÉTODO}

\section{Muestra}

La muestra estuvo conformada por 275 estudiantes de $1^{\circ}$ y $4^{\circ}$ año de las facultades de Ingeniería y Humanidades de la Universidad Católica del Norte (Sede Antofagasta), Chile. Sus edades comprendían entre los 17 y 32 años, con una media de 20.63 (D.T.= 2.85). En cuanto al sexo participaron 160 hombres, que corresponden al $58.4 \%$ y 114 mujeres que correspondan al $41.6 \%$.

\section{Instrumentos}

El instrumento estuvo compuesto por tres secciones, la primera de ellas incluía preguntas destinadas a caracterizar la muestra a través de antecedentes sociodemográficos personales y de la familia de procedencia, así como el historial académico previo. La segunda sección contenía el Cuestionario de Metas Académicas (CMA) de Hayamizu y Wainer (1991). Este cuestionario consta de 20 ítems, el cual fue presentado en una escala Likert de 6 puntos. Posee dos sub escalas que evalúan la presencia de metas de dominio y metas de rendimiento respectivamente. Las bondades psicométricas del CMA han sido ampliamente demostradas (González y cols. 2002; Navas y cols. 2002; Navas y Sampascual, 2008). En nuestro estudio esta escala alcanzó una fiabilidad de .87 para la escala total.

La tercera sección estaba compuesta por la escala EFEC (Escala de Factores Estructurales de la Clase, la cual corresponde a una traducción y adaptación del modelo de evaluación de 4 de los factores estructurales de la clase TARGET system de Ames (1992a). Este instrumento busca indagar la percepción de los estudiantes acerca de la presencia de los factores estructurales de la clase que fomentan la adquisición de metas de dominio, planteados por Ames, anteriormente descritos. Este cuestionario quedó conformado por 14 ítems presentados en escala Likert de 6 puntos.

\section{Procedimiento}

El instrumento fue presentado a los participantes como parte de un estudio que tenía como propósito conocer algunas características de los estudiantes a la hora de aprender. Fue aplicado de forma colectiva en el aula, en cada uno de los cursos, en los 20 últimos minutos de clase, previo acuerdo con el profesor.

\section{RESULTADOS}

\section{Estructura factorial}

En un primer momento, para identificar la estructura interna, hemos realizado un Análisis Factorial Confirmatorio buscando determinar las dimensiones subyacentes que expliquen la red de correlaciones presentes en nuestros datos.

Previo al proceso de factorización y con el propósito de determinar el número de factores existentes en nuestros datos 
hemos realizado un Análisis Paralelo (AP) (Horn, 1965). Este análisis propone comparar los autovalores encontrados empíricamente en los datos reales con los obtenidos a partir de múltiples matrices de variables no correlacionadas considerando el mismo número de variables que los datos empíricos y en el mismo tamaño de muestral. SE retienen los componentes empíricos con autovalores superiores a los de la matriz son retenidos. Los resultados se muestran en la tabla 2.
Como es posible observar el análisis paralelo sugiere la retención de sólo un factor, el cual obtienen un autovalor superior al autovalor aleatorio. Por el contrario la regla K-1 sugiere la retención de dos factores, ya que a partir del tercer factor los autovalores son inferiores a 1. Dada que ninguna de las dos soluciones es completamente satisfactoria desde el punto de vista teórico hemos realizado un análisis factorial confirmatorio comparando varios modelos alternativos con el fin de encontrar la estructura factorial que mejor se ajuste a los datos.

TABLA 2.

Análisis Paralelo

\begin{tabular}{rrrr}
\hline \multicolumn{2}{c}{$\begin{array}{c}\text { Autovalores } \\
\text { reales }\end{array}$} & $\begin{array}{c}\text { Madia autovalores } \\
\text { aleatorios }\end{array}$ & $\begin{array}{c}\text { P95 autovalores } \\
\text { aleatorios }\end{array}$ \\
\hline 1 & 7.631 & 1.403 & 1.487 \\
2 & 1.189 & 1.305 & 1.370 \\
3 & .864 & 1.321 & 1.286 \\
4 & .730 & 1.169 & 1.220 \\
5 & .631 & 1.113 & 1.154 \\
6 & .529 & 1.061 & 1.102 \\
7 & .482 & 1.011 & 1.052 \\
8 & .455 & .962 & 1.001 \\
9 & .334 & .915 & .953 \\
10 & .305 & .867 & .906 \\
11 & .264 & .818 & .858 \\
12 & .232 & .769 & .809 \\
13 & .191 & .716 & .760 \\
14 & .157 & .652 & .707 \\
\hline
\end{tabular}

Para el análisis del modelo confirmatorio hemos utilizado el método de Máxima Verosimilitud sobre la matriz de covarianzas, utilizando el programa AMOS 18. Para confirmar el modelo de medida sometimos a prueba una serie de modelos con el fin de determinar cuál de ellos presenta el mejor ajuste. Los índices de bondad de ajuste que hemos considerado para evaluar la bondad del modelo de medición fueron: $x 2$, X2/d.f., RMSEA (Root Mean Square Error of Aproximation), los índices incrementales (CFI, NFI y NNFI), y los índices basados en las discrepancias de las matrices GFI. 
TABLA 3.

Análisis factorial confirmatorio. Medidas de ajuste global

\begin{tabular}{lcccccccccc}
\hline & $X^{2}$ & $g l$ & $\Delta X^{2}$ & $\Delta g l$ & $X^{2} / g l$ & RMSEA & NFI & TLI & CFI & GFI \\
\hline 1) 1 factor & $420.199^{*}$ & 77 & & & 5.457 & .129 & .835 & .834 & .860 & .811 \\
2) 2 factores correlacionados & $279.330^{*}$ & 76 & $140.869^{*}$ & 1 & 3.675 & .100 & .892 & .902 & .918 & .872 \\
$\begin{array}{l}\text { 3) } 2 \text { factores de 2 }{ }^{\circ} \text { orden y } 4 \\
\text { de } 1^{\circ} \text { orden }\end{array}$ & $196.008^{*}$ & 72 & $83.322^{*}$ & 4 & 2.722 & .080 & .923 & .936 & .949 & .911 \\
4) 4 factores relacionados & $196.007^{*}$ & 71 & .001 & 1 & 2.761 & .081 & .923 & .935 & .949 & .911 \\
\hline
\end{tabular}

* La probabilidad es inferior a .05

Como es posible observar, se ha sometido a prueba una serie de modelos. Se presentan los resultados de ajuste del modelo de un factor propuesto por AP, el modelo de 2 factores correlacionadas, sugerido por la regla K-1. En ambos encontramos problemas de ajuste. A pesar que el modelo de dos factores presenta mejoras importantes en el ajuste, algunos de sus índices no alcanzan los criterios mínimos aceptables.

Dado que estos resultados no logran ser satisfactorios hemos optado por contrastar el ajuste de dos modelos coherentes con la propuesta teórica de Ames (1992). La tabla 3 nos muestra el modelo 3 , que corresponde a 4 factores propuestos con dos factores de $2^{\circ}$ orden y el modelo 4 , que propone 4 factores correlacionados.

Como es posible observar ambos presentan índices de ajuste medianamente aceptables (NFI, TLI, CFI y GFI .90 y RMSEA $=.08$ ). La diferencia del ajuste entre ambos modelos no llega a ser significativo $\left(\Delta X^{2}{ }_{1}=.001 ; p>.05\right)$.

Por lo tanto, al no existir diferencias significativas en el nivel de ajuste de ambos modelos hemos optado por el modelo 3, más parsimonioso (posee un grado de libertad más). La estructura del modelo final es presentada en la figura 1.

Es así que se presentan, desde el punto de vista de los estudiantes, dos grandes dimensiones de evaluación de la estructura de la clase. Por un lado el tipo de Relación Pedagógica o Relación de Aprendizaje, que se establece entre profesor y estudiante. Esta es expresada a través de las Actividades de Aprendizaje desarrolladas por el profesor y el tipo de Autoridad que este despliega. Por otro lado, el proceso de Seguimiento, sería otra importante dimensión en la evaluación de la clase, la cual se concreta a través del tipo de evaluación implementada por el profesor y el sistema de Refuerzo utilizado para promover el aprendizaje de los estudiantes.

\section{Fiabilidad}

Para determinar la fiabilidad, hemos utilizado la medida de consistencia interna Alfa de Cronbach. Este coeficiente logró un valor de .94 para el total de la escala, y valores de $.82, .86, .79$ y .91 para las subescalas Actividades de aprendizaje, Autoridad, Sistema de refuerzo y Sistema de evaluación, respectivamente. Este resultado muestra que la escala posee una alta consistencia interna y estabilidad en la medida, demostrándose altamente fiable. 


\section{Correlaciones}

Para determinar en qué medida estos factores estructurales logran estar relacionados con las metas académicas, hemos calculado las correlaciones de Pearson entre las puntuaciones de cada uno de los factores estructurales, de $1^{\circ}$ y $2^{\circ}$ orden, así como la puntuación total en la escala y la puntuación en las dos metas. Los resultados se pueden observar en la tabla 3.

Las correlaciones nos muestran que los factores estructurales alcanzaron correlaciones moderadas pero estadísticamente significativas con la puntuación de los estudiantes en la subescala Metas de Dominio. Por el contrario, la subescala Metas de Logro no obtuvo correlaciones significativas con ninguno de los factores estructurales ni con la puntuación total.

Estos datos nos demuestran que los factores estructurales corresponden a variables relacionadas con las metas de dominio de los estudiantes. Po tanto promover la adquisición de metas de dominio, a través de la intervención en el aula, estaría relacionado con la adquisición de metas de dominio, y con ellos, el rendimiento académico.

\section{FIGURA 1.}

Análisis factorial confirmatorio. Path Diagram del modelo final.

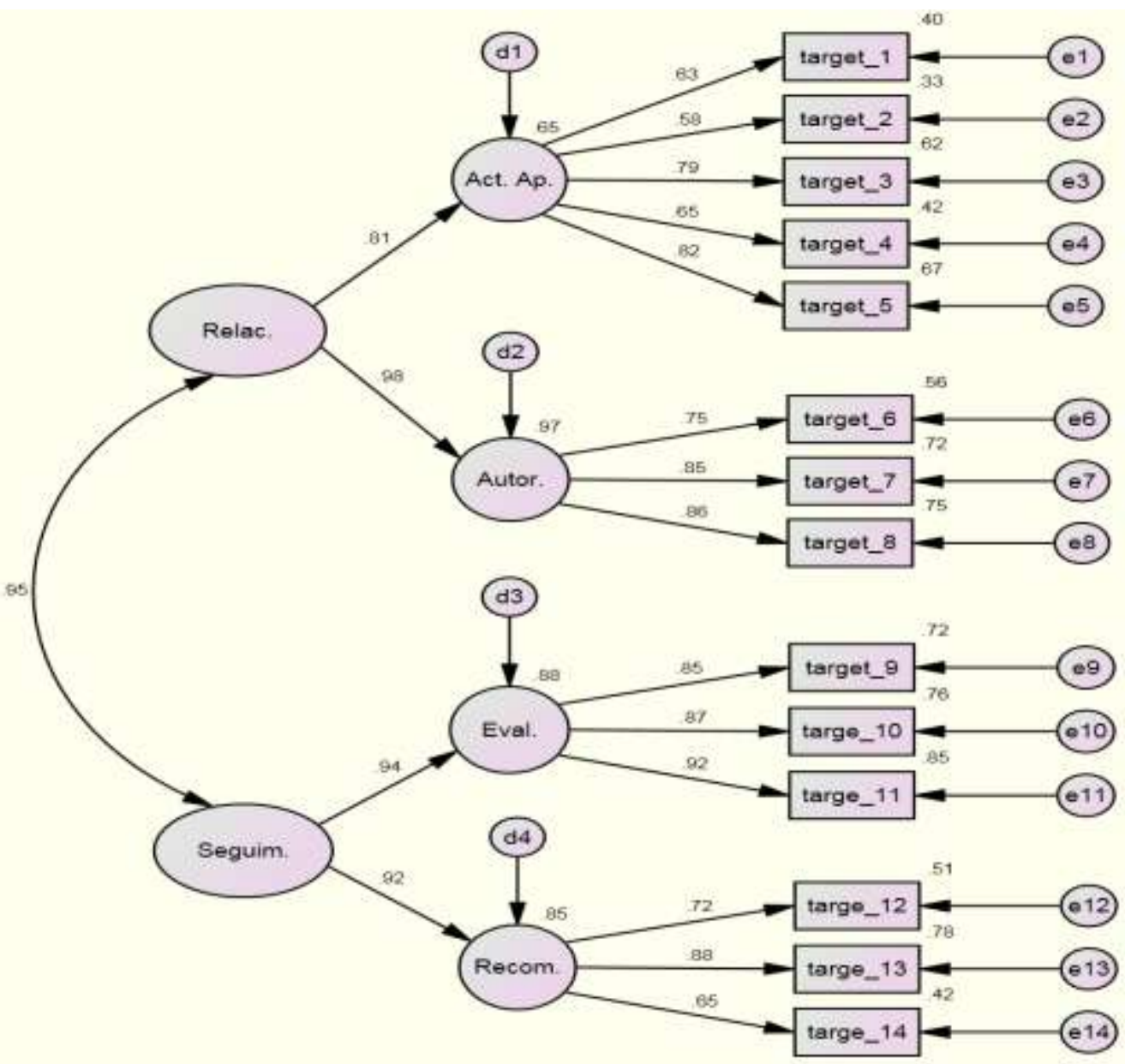

FIGURA 1. Modelo de medida luego del análisis factorial confirmatorio. El modelo presenta 4 factores de primer orden que corresponde al 4 dimensiones propuestas por Ames (1992) y 2 factores de $2^{\circ}$ orden. El primero de ellos es llamado "Relación de aprendizaje", que agrupa a las dimensiones Actividades de Aprendizaje y Autoridad, y el segundo "Seguimiento del Aprendizaje" que agrupa a los factores Sistema de refuerzo y Sistema de evaluación. 
TABLA 4.

Correlaciones entre "Factores estructurales de la clase" y Orientación de metas

\begin{tabular}{|c|c|c|c|c|c|c|c|}
\hline & \multicolumn{4}{|c|}{ Factores de $1^{0}$ orden } & \multicolumn{2}{|c|}{ Factores de $2^{\circ}$ orden } & \multirow[t]{2}{*}{ Total } \\
\hline & Actividad & Autoridad & Evaluación & Recompensa & $\begin{array}{l}\text { Relación de } \\
\text { aprendizaje }\end{array}$ & $\begin{array}{c}\text { Seguimiento } \\
\text { del aprendizaje }\end{array}$ & \\
\hline Metas dominio &, $\left.392^{(*}\right)$ &, $\left.319^{(* *}\right)$ & ,292(**) &, $\left.308^{* *}\right)$ &, $389\left(^{(*}\right)$ &, $\left.340^{(* *}\right)$ &, $387(* *)$ \\
\hline Metas de logro & 100 & 061 & 061 & ,030 & 089 & 054 & ,079 \\
\hline $\begin{array}{l}\text { Actividad } \\
\text { aprendizaje }\end{array}$ & 1 &, $\left.699{ }^{(* *}\right)$ &, $576\left(^{* *}\right)$ &, $\left.639{ }^{* *}\right)$ &, $937\left(^{* *}\right)$ &, $670\left(^{* *}\right)$ &, $852\left(^{* *}\right)$ \\
\hline Autoridad & & 1 &, $\left.730^{* \star}\right)$ &, $788\left(^{* \star}\right)$ &, $\left.905^{(*}\right)$ &, $\left.812^{(* *}\right)$ &, $908\left(^{* \star}\right)$ \\
\hline Evaluación & & & 1 &, $\left.7499^{* \star}\right)$ &, $\left.700{ }^{* \star}\right)$ &, $905\left(^{* \star}\right)$ &, $843\left(^{* \star}\right)$ \\
\hline Recompensa & & & & 1 &, $\left.765^{(*}\right)$ &, $943\left(^{* *}\right)$ &, $898\left(^{* *}\right)$ \\
\hline $\begin{array}{l}\text { Relación de } \\
\text { aprendizaje }\end{array}$ & & & & & 1 &, $796\left(^{* \star}\right)$ &, $\left.9511^{* *}\right)$ \\
\hline $\begin{array}{l}\text { Seguimiento } \\
\text { del aprendizaje }\end{array}$ & & & & & & 1 &, $944(* *)$ \\
\hline Total & & & & & & & 1 \\
\hline
\end{tabular}

\section{DISCUSIÓN}

Los análisis realizados han demostrado que la Escala de Factores Estructurales de la Clase, es coherentemente con la teoría de Ames (1992a), transformándose en un instrumento fiable, con un alto grado de consistencia interna.

En análisis de la estructura interna mostro la existencia de cuatro factores, estructura coherente con la propuesta de Ames, agrupados en dos factores de segundo orden. Estos resultados nos sugieren la existencia de dos grandes dimensiones que agruparían las características, percibidas por los estudiantes, que promueven la adquisición de Metas de Dominio.

Esta última idea es reforzada por las correlaciones encontradas entre los factores estructurales y las orientaciones de metas, lo cual es coherente con la propuesta de Ames. Un elemento importante de este marco comprensivo es el énfasis existe, no en la estructura objetiva de la clase, sino más bien en la percepción subjetiva que el estudiante hace de ella.

De hecho, el énfasis de este modelo comprensivo recae en el significado que el estudiante le atribuye a las estructuras. Es por ello que medidas de esta naturaleza se convierten en una contribución al abordaje de la dinámica interactiva al interior de la sala de clases.

Sin lugar a duda, la dinámica en la que se desarrolla la clase, en tanto sistema social complejo, nos obliga como educadores, no solo poner atención en aspectos individuales de los estudiantes, sino que también en elementos relacionales o situacionales del ambiente que puedan influir en como el estudiante se aproxima al aprendizaje.

Es así que la teoría de Orientación de metas, y particularmente los factores estructurales de la clase, nos entregan un marco, desde un punto de vista sociocognitivo, desde el cual comprender y abordar la motivación al aprendizaje de 
parte de los estudiantes. Por tanto, el instrumento que hemos presentado se transforma en una oportunidad de poder abordar estas variables situacionales.

Considerando esto, creemos que este instrumento es una herramienta adecuada para evaluar los factores estructurales de la clase, al mostrar una confiabilidad optima, una estructura factorial coherente que asegura la validez de constructo, y correlaciones significativas con las metas de dominio de los estudiantes, lo que evidencia en relación a la validez de criterio.

Es así que este instrumento se transforma en una medida valida y confiable para medir la percepción que tienen los estudiantes de las características del ambiente de aprendizaje.

Por tanto, creemos que este instrumento es una contribución tanto para el estudio de la motivación académica, desde la perspectiva de la orientación de metas, como para la evaluación de intervenciones e innovaciones educativas por parte del profesor, enfocadas en estos factores estructurales.

\section{REFERÊNCIAS}

Ames, C. (1984b). Competitive, cooperative, and individualistic goal structures: A motivational analysis. In R. Ames \& C. Ames (Eds.), Research on motivation in education: Vol 1. Student motivation. New York: Academic Press.

Ames, C. (1992a). Classrooms: Goals, Structure, and Student Motivation. Journal of Educational Psychology, 84(3), 261271.

Ames, C. (1992b). Achievement goals and classroom motivational climate. In $\mathrm{J}$. Meece \& D. Schunk (Eds.) Students' perceptions in the classroom (pp.327-348). Hillsdale, NJ: Erlbaum.

Ames, C. \& Archer, J. (1988). Achievement Goals in the Classroom: Student Learning Strategies and Motivation Processes.
Journal of Educational Psychology, 80(3). 260-267.

Dweck, C.S. 81986). Motivational process affecting learning. American Psychologist, 41, 1040-1048.

Dweck, C, Y Leggett, E. (1986). A Social Cognitive approach to motivation and personality. Psychological Review, 95, 256-273.

Elliot, E. \& Dweck, C. (1988). An Approach to the motivation and Achievement. Journal of Personality and Social Psychology, 54, 512

Harackievicz, J. M., Barron, K., Tauer, J., Carter, S. \& Elliot, A. (2000). Short-term and Longterm consequences of Achievement Goals: Predicting Interest and Performance over time. Journal of Educational Psychology, 92, 316-330.

González-Cabanach, R. Valle, A. Nuñez, C. \& Gonzalez-PIEDA, J. (1996). Una Aproximación Teórica al Concepto de Metas Académicas. Psicothema, 8 (1), 4561.

Gonzalez, C. Torregrosa, G. \& NAVAS, L. (2002). Un Análisis de las Metas en Situación de Aprendizaje para el Alumnado de Primaria y Secundaria Obligatoria. Revista Española de Orientación y Psicpedagogía, 13(1), 69-87.

Grant, H. \& Dweck, C. (2003). Clarifying Achievement Goal and Their Impact. Journal of Personal and Social Psychology, $85,541-517$.

Hayamizu, T. \& Weiner, B. (1991). A test Dweck's Model of Achievement Goals as Related to Perceptions of Ability. Journal of Experimental Education, 59, 226-234.

Horn, J. L. (1965). A rationale and test for the number of factors in factor analysis. Psychometrika, 30, 179-185.

Maher, M.L. \& Nicholls, J.G. (1980). Culture and achievement motivation: A second look. In N. Warren (Ed.), Studies in cross cultural psychology. San Diego, CA: Academic Press.

Martinez, M. Hernandez, M. V. \& M. Hernandez, M. J. (2006). Psicometría. Ed. Alianza. Madrid.

Meece, Anderman \& Anderman (2006). Classroom Goal Structure, Student Motivation and Academic Achievement. Annual Review of Psychology, 57. 478503. 
Miller, R., Greene, B., Montalvo, G., Ravindran, B., \& Nichols, J. (1996). Engagement in academic work: the role of learning goals, future consequences, pleasing others, and perceived ability. Contemporary Educational Psychology, 21, 388-422.

Navas, L. González, C. \& Torregrosa, G. (2002). Metas de Aprendizaje: Un Análisis Transversal de las Estructuras Factoriales que Presentan. Revista de Psicología General y Aplicada., 55(2), 553-564.

Navas, L. \& Sampascual, G. (2008). Un Análisis Exploratorio y Predictivo Sobre la Orientación de Metas y Sobre el Contenido de las Metas de los Estudiantes. Horizontes educacionales, 13(1), 23-34.

Nicholls, J. G. (1984). Achievement motivation: Conceptions of ability, subjective experience, task choice, and performance. Psychological Review, 91(3), 328-346.

Pintrich, P.R. (2000). Multiples Goal, Multiples Pathway: The Role of Goal Orientation in Learning and Achievement. Journal of Educational Psychology, 92, 544-555.

Valle, A., Gonzalez-Cabanach,R. Rodriguez, S. Nuñez, J. C. \& Gonzalez-Pieda, J. A. (2006). Metas Académicas, estrategias cognitivas y estrategias de regulación del Estudio. Psicothema, 18,(2), 165-170.

Weiner, B. (1986). An Atributional theory of motivation and emotion. Springer-Verlag. New York.

Wolters, C. (2004). Advancing Achievement Goal Theory: Using Goal Structures and Goal Orientations to Predict Students' Motivation, Cognition and Achievement. Journal of Educacional Psychology, 96, 236-250.

Wentzel, K. R. (2000). Social-Motivational Processes and Interpersonal Relationship Students' Academic Success. Journal of Educational Psychology 91, 76-97. 
ANEXO

\begin{tabular}{l} 
Escala de Factores Estructurales de la Clase \\
\hline 1. Las actividades de aprendizaje fueron variadas \\
2. Las actividades de aprendizaje fueron desafiantes \\
3. Las actividades de aprendizaje fueron estimulantes \\
4. Las actividades de aprendizaje fueron desarrolladas de manera organizada a lo largo del curso \\
5. Las actividades de aprendizaje promovieron el interés hacia las materias desarrolladas \\
6. El profesor entregó responsabilidades al grupo curso sobre el propio proceso de aprendizaje \\
7. El profesor dio posibilidades a los estudiantes de tomar decisiones con respecto al proceso de enseñanza-aprendizaje \\
8. El profesor estimuló a los estudiantes a tomar un rol activo en el proceso de enseñanza-aprendizaje \\
9. El profesor incentivó y recompensó el esfuerzo de los estudiantes \\
10. El profesor asistió y acompañó el progreso de los estudiantes \\
11. El profesor estimuló a los estudiantes a superarse constantemente \\
12. El profesor utilizó diferentes estrategias de evaluación \\
13. El profesor retroalimentó la evaluación a los estudiantes comentando las fortalezas e identificando los aspectos a \\
mejorar \\
14. La evaluaciones se centraron en el dominio de habilidades más que en la memorización
\end{tabular}

MATRIZ DE VARIANZAS - COVARIANZAS OBSERVADAS

\begin{tabular}{|c|rrrrrrrrrrrrrr|r||}
\hline items & 14 & 13 & 12 & 11 & 10 & 9 & 8 & 7 & 6 & 5 & 4 & 3 & 2 & 1 \\
\hline 14 & 1.856 & & & & & & & & & & & & \\
13 & 1.252 & 2.386 & & & & & & & & & & & \\
12 & .939 & 1.436 & 2.265 & & & & & & & & & & \\
11 & 1.045 & 1.693 & 1.361 & 2.295 & & & & & & & & & \\
10 & .962 & 1.452 & 1.289 & 1.828 & 2.283 & & & & & & & & \\
9 & .941 & 1.622 & 1.328 & 1.895 & 1.887 & 2.673 & & & & & & & \\
8 & .929 & 1.569 & 1.238 & 1.581 & 1.459 & 1.508 & 2.214 & & & & & & \\
7 & .954 & 1.487 & 1.207 & 1.609 & 1.434 & 1.651 & 1.702 & 2.333 & & & & & \\
6 & .764 & 1.215 & 1.010 & 1.319 & 1.226 & 1.263 & 1.325 & 1.302 & 1.940 & & & & \\
5 & .863 & 1.085 & .788 & 1.093 & 1.023 & 1.152 & 1.060 & 1.084 & 1.050 & 1.846 & & & \\
4 & .770 & .704 & .631 & .878 & .770 & .874 & .929 & .879 & .954 & 1.010 & 1.631 & & \\
3 & .675 & .900 & .724 & .998 & .931 & 1.076 & .944 & 1.108 & .773 & 1.231 & .743 & 1.723 & \\
2 & .466 & .686 & .481 & .747 & .564 & .531 & .594 & .638 & .620 & .623 & .470 & .643 & 1.213 \\
1 & .389 & .757 & .667 & .754 & .726 & .826 & .789 & .874 & .587 & .689 & .563 & .811 & .698 & 1.376 \\
\hline
\end{tabular}

